\title{
Effect of Different Near-Wall Treatments on Indoor Airflow Simulations
}

\author{
N. El Gharbi ${ }^{1,3}$, R. Absi ${ }^{2 \dagger}$, A. Benzaoui ${ }^{3}$ \\ ${ }^{1}$ Center for Renewable Energy Development, Po. Box 62 Bouzareah 16340 Algiers, Algeria \\ ${ }^{2}$ EBI, Inst. Polytech. St-Louis, PRES UPGO - Université Paris Grand Ouest, 32 Boulevard du Port, 95094, Cergy- \\ Pontoise Cedex, France \\ ${ }^{3}$ University of Sciences and Technology Houari Boumediene(USTHB), Po. Box 32 El Alia Bab Ezzouar 16111 \\ Algiers, Algeria \\ †Corresponding Author Email: rafik.absi@yahoo.fr
}

(Received October 30, 2010; accepted July 4, 2011)

\begin{abstract}
Airflow simulation results depend on a good prediction of near wall turbulence. In this paper a comparative study between different near wall treatments is presented. It is applied to two test cases in building: (1) the first concerns flow through a long corridor which is similar to that in a fully developed plane channel. Simulation results are compared to direct numerical simulation (DNS) data of Moser et al. (1999) for $R e_{\tau}=590$ (where $R e_{\tau}$ denotes the friction Reynolds number defined by friction velocity $u_{\tau}$, kinematics viscosity $v$ and the channel half-width $\delta$ ); (2) the second case is a benchmark test for room air distribution. Simulation results are compared to experimental data obtained with laser-Doppler anemometry (Nielsen, 1990). Simulations were performed with the aid of CFD code Fluent (2005). Near wall treatments available in Fluent were tested: Standard Wall Functions, Non Equilibrium Wall Function and Enhanced Wall Treatment. In each case, suitable meshes with adequate position of the first near-wall node are needed. Results of near-wall mean stream wise velocity $u^{+}$and turbulent kinetic energy $k^{+}$profiles are presented, variables with the superscript of + are those non dimensional by the wall friction velocity $u_{\tau}$ and the kinematic viscosity $v$.
\end{abstract}

Keywords: CFD, Airflow, Turbulence, Simulation, Near-Wall treatment, Channel, Room.

\section{NOMENCLATURE}

$C_{1}, C_{2}$ Constant of empirical turbulence model for $k-\varepsilon$ one

$G_{k} \quad$ Generation of $k,\left(\mathrm{Nm}^{-2}\right)$

$H \quad$ High, (m)

$k$ Turbulent kinetic energy, $\left(\mathrm{m}^{2} \mathrm{~s}^{-2}\right)$

$L \quad$ Width, (m)

$R_{i j} \quad$ Reynolds stress tensor, $\left(\mathrm{kg} \mathrm{m}^{-1} \mathrm{~s}^{-2}\right)$

$R e_{\tau} \quad$ Friction Reynolds number

$u_{0} \quad$ Inlet velocity, $\left(\mathrm{m} \mathrm{s}^{-1}\right)$

$u_{i}^{\prime}, u_{j}^{\prime}$ Arbitrary fluctuating velocity

component, $\left(\mathrm{m} \mathrm{s}^{-1}\right)$

$u_{\tau} \quad$ Friction velocity, $\left(\mathrm{m} \mathrm{s}^{-1}\right)$

$x \quad$ Axial coordinate, $(\mathrm{m})$

$x_{i}, x_{j}$ Arbitrary direction, (m)

$u_{\tau} \quad$ Friction velocity, $\left(\mathrm{m} \mathrm{s}^{-1}\right)$

$y \quad$ Radial coordinat, $(\mathrm{m})$

$y^{+} \quad$ No-dimensional wall coordinate

\section{Greek symbols}

$\delta_{i j} \quad$ Kronecker delta

$\varepsilon \quad$ Turbulent energy dissipation rate, $\left(\mathrm{m}^{2} \mathrm{~s}^{-3}\right)$

$\mu \quad$ Dynamic viscosity, $\left(\mathrm{kgm}^{-1} \mathrm{~s}^{-1}\right)$

$\mu_{t} \quad$ Turbulent viscosity , $\left(\mathrm{kgm}^{-1} \mathrm{~s}^{-1}\right)$

$v \quad$ Kinematics viscosity, $\left(\mathrm{m}^{2} \mathrm{~s}^{-1}\right)$

$\rho \quad$ Fluid density, $\left(\mathrm{kgm}^{-3}\right)$

$\tau_{i j} \quad$ Turbulent stress tensor, $\left(\mathrm{m}^{2} \mathrm{~s}^{-2}\right)$

$\tau_{w} \quad$ Wall shear stress, $\left(\mathrm{kg} \mathrm{m}^{-1} \mathrm{~s}^{-2}\right)$

\section{Abbreviation}

CFD Computational Fluid Dynamics

DNS Direct Numerical Simulation

EWT Enhanced Wall Treatment

NEWF Non-Equilibrium Wall Function

RMS Root Mean Square

SWF Standard Wall Function

TKE Turbulent Kinetic Energy 


\section{INTRODUCTION}

Most people spend the majority of their time indoors, often in shared spaces, so the expectations of the occupant for a thermally comfortable indoor climate have risen. For this reason tools are required to determine and predict the flow characteristics in the early design phase. The CFD method is often employed.

For predicting room air flow, the standard $k$ - $\varepsilon$ turbulence model has enjoyed the greatest usage (Murakami et al. (1987), Nielsen (1989), Chen et al. (1992), Weathers (1992), Haghighat et al. (1992), Chen (1995), El Gharbi (2007), Sumon et al. (2008), Bahlaoui et al. (2011), El Gharbi et al. (2012)). However this model is only valid for fully-developed turbulence, the flow is not solved up the wall. In addition, the wall is the most common boundary encountered in these confined fluid flow problems. Therefore, to simulate this region the selection of appropriate near-wall treatment methods is very important for obtaining reliable prediction results of airflows simulation. The first near wall treatments was developed with $k-\varepsilon$ model, we quoted: Spalding (1961), Wolfstein (1969), Launder et al. (1974), Chen et al. (1988), Jongen (1992), Kim et al. (1995).

This investigation studied two typical indoor airflows: (1) a flow in a fully developed plane channel, assimilated to flow through a long corridor, (2) a forced convection flow in a ventilated room, a benchmark test for 2D room air distribution. Fluid flow near a solid wall as well as the characteristics of turbulent flow near such structures is considered. Simulations will be performed with the aid of the commercial CFD code Fluent (2005). All different near wall treatments available in Fluent will be tested: Standard Wall Functions, Non Equilibrium Wall Function and Enhanced Wall Treatment. We will investigate both effects of meshes and position of the first near-wall node.

For the first test case simulations results are compared to direct numerical simulation (DNS) data of Moser et al. (1999) for $R e_{\tau}=590$ (where $R e_{\tau}$ denotes the friction Reynolds number defined by friction velocity $u_{\tau}$, kinematic viscosity $v$ and the channel half-width $\delta$ ). Then, for the second test case, the simulation results are compared with experimental data obtained with laserDoppler anemometry (Nielsen, 1990). This one is use to measure velocity and velocity fluctuation.

\section{Model EQuations}

\section{A. Governing Equations}

In this study, airflow is modeled using the standard $k-\varepsilon$ model. The governing equations are:

Mass conservative equation:

$$
\frac{\delta\left(\rho u_{i}\right)}{\delta x_{i}}=0
$$

Momentum conservation equation: $\frac{\delta\left(\rho u_{i} v_{j}\right)}{\delta x_{i}}=-\frac{\delta p}{\delta x_{i}}+\frac{\delta \tau_{i j}}{\delta x_{j}}+\frac{\delta R_{i j}}{\delta x_{j}}$

where $\tau_{i j}$ is the viscous stress tensor and $R_{i j}$ is the turbulent Reynolds stress tensor

$$
\begin{aligned}
& \tau_{i j}=\mu\left(\frac{\delta u_{i}}{\delta x_{j}}+\frac{\delta u_{j}}{\delta x_{i}}\right)-\frac{2}{3} \mu\left(\frac{\delta u_{k}}{\delta x_{k}}\right) \delta_{i j} \\
& R_{i j}=-\rho \overline{u_{i}^{\prime} u_{j}^{\prime}}=\mu_{t}\left(\frac{\delta u_{i}}{\delta x_{j}}+\frac{\delta u_{j}}{\delta x_{i}}\right)-\frac{2}{3} \rho k \delta_{i j}
\end{aligned}
$$

Turbulent kinetic energy:

$\rho u_{i} \frac{\delta k}{\delta x_{i}}=\frac{\delta}{\delta x_{i}}\left[\left(\mu+\frac{\mu_{t}}{\sigma_{k}}\right) \frac{\delta k}{\delta x_{i}}\right]+G_{k}-\rho \varepsilon$

Dissipation rate:

$$
\begin{aligned}
& \rho u_{i} \frac{\delta \varepsilon}{\delta x_{i}}=\frac{\delta}{\delta x_{i}}\left[\left(\mu+\frac{\mu_{t}}{\sigma_{k}}\right) \frac{\delta \varepsilon}{\delta x_{i}}\right]+ \\
& C_{1}\left(\frac{\varepsilon}{k}\right) G_{k}-C_{2} \rho \frac{\varepsilon^{2}}{k} \\
& \mu_{t}=C_{\mu} \rho\left(\frac{k^{2}}{\varepsilon}\right) \\
& G_{k}=\mu_{t}\left(\frac{\delta u_{i}}{\delta x_{j}}+\frac{\delta u_{j}}{\delta x_{i}}\right) \frac{\delta u_{j}}{\delta x_{i}}-\frac{2}{3} \rho k \delta_{i j} \frac{\delta u_{j}}{\delta x_{i}}
\end{aligned}
$$

\section{B. Near-wall Treatments}

Close to the wall, the flow is influenced by viscous effects. The mean velocity field is affected through the no-slip condition that has to be satisfied at the wall. Toward the outer part of the near-wall region, however, the turbulence is rapidly augmented by the production of turbulence kinetic energy due to the large gradients in mean velocity.

Therefore, accurate representation of the flow in the near-wall region determines successful predictions of wall-bounded turbulent flows. For that and because most $k-\varepsilon$ and RSM turbulence models will not predict correct near-wall behavior if integrated down to the wall, special near-wall treatment is required.

\section{Fluent near-wall treatments:}

Fluent offers two approaches based on the classical theory describing the flow near-walls in turbulent flows, Fluent (2005), Fig. 1:

a. The first one is a semi-empirical approach, and uses the so called "wall function" to bridge the viscosity affected region between the wall and the fully turbulent region. The viscous sublayer and buffer layer region are not resolved. Therefore, the near-wall mesh may be relatively coarse, the first grid point off the wall must be positioned in the log law region at $y^{+}>30$ (the distance being measured in wall units $y^{+}=y u_{t} v$, where $u_{\tau}$ is the friction velocity). This approach is justified for 
industrial flows with high Reynolds numbers, because it saves computational time and it is sufficiently precise. There are two options for semi-empirical approach use in Fluent code. The first "Standard Wall Function" (Launder et al. 1974) is presented as default in Fluent. It assumes equilibrium between the production and dissipation of turbulent kinetic energy. The second "NonEquilibrium Wall Function" (Kim et al. 1995) may be selected by the user. It does not assume this equilibrium, but allows differing production and dissipation, as may be the case for flows where there is separation and reattachment or severe pressure gradients (Fluent 2005).

b. The second approach combines a two layer model (where the viscosity affected near-wall region is completely resolved, along the way to the viscous sublayer), together with enhanced wall functions. Generally, it requires a very fine near-wall mesh. The first grid point off the wall must be from $y^{+} \approx 1$. This approach is more suited for lowReynolds number flows with complex nearwall phenomena. Although it obviously requires a greater amount of computational resources. (Fluent 2005).

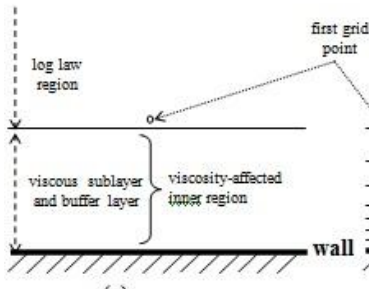

(a)

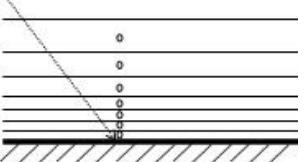

(b)
Fig. 1. Schematics of (a), the wall function approach and (b) the two-layer approach

\section{Test CASES}

Airflow simulations with different near-wall treatments are applied to two test cases:

\section{A. Channel flow}

The first test case is the plane fully developed channel flow similar to flow through a long corridor in a building, Fig. 2. Simulations results are validated by DNS data of Moser et al. (1999) for $\operatorname{Re}_{\tau}=590$.

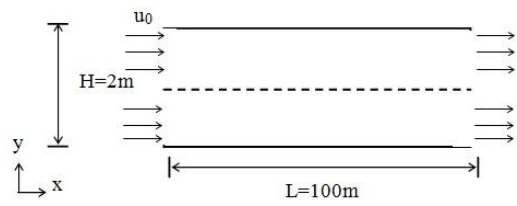

Fig. 2. Presentation of the channel flow

\section{B. Room air distribution}

The second test case is a benchmark test (Annex 20, Nielsen, 1990) for a room air distribution, Fig. 3. The simulation results are validated by experimental data obtained with Laser-Doppler Anemometry.

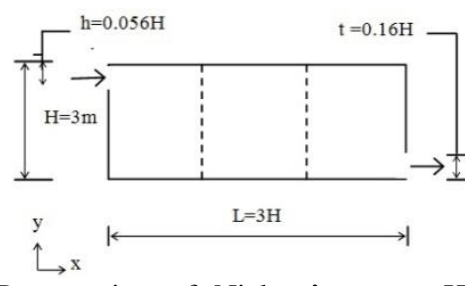

Fig. 3. Presentation of Nielsen's room, $H=3 m$ and $\mathrm{L}=9 \mathrm{~m}$.

\section{RESUltS AND DISCUSSIONS}

\section{A. Effect of Mesh and First Grid Point}

To captures boundary layer properly, the mesh should be correctly generated. For turbulent flows, calculation of the $y^{+}$value of the first node point helps in doing that. This dimensionless distance is defined as:

$y^{+}=\frac{u_{\tau} y}{v}$

where $u_{\tau}$ is the friction velocity defined as $\sqrt{\frac{\tau_{w}}{\rho}}$ and $\tau_{w}$ is the wall shear stress.

For that and because the wall distance $y^{+}$is involved in the selection of the appropriate near-wall treatment, we do a grid test for only mesh in $y$ direction. The geometry of a fully developed plane channel is chosen (first test case) and eight different mesh sizes are applied to select the appropriate mesh size that adapt with near-wall treatment (wall functions or near-wall modeling). This is achieved by refining the mesh, with particular attention to the first grid point off the wall. Table 1 shows selected computational mesh and the corresponding wall $y^{+}$values.

Table 1 Different mesh

\begin{tabular}{lccc}
\hline & $\begin{array}{c}\text { mesh size } \\
(x \times y)\end{array}$ & first $y^{+}$ & mesh type \\
\hline mesh 1 & $(500 \times 10)$ & 59 & regular \\
mesh 2 & $(500 \times 14)$ & 42.142874 & regular \\
mesh 3 & $(500 \times 19)$ & 31.052644 & regular \\
mesh 4 & $(500 \times 28)$ & 21.071437 & regular \\
mesh 5 & $(500 \times 57)$ & 10.350901 & regular \\
mesh 6 & $(500 \times 76)$ & 7.763161 & regular \\
mesh 7 & $(500 \times 57)$ & 1.3467045 & exponential law \\
mesh 8 & $(500 \times 76)$ & 1.0026873 & exponential law \\
\hline
\end{tabular}

Non-dimensional mean stream wise velocity profiles scaled by the wall velocity $u^{+}=u / u_{t}$ and nondimensional profiles of turbulent kinetic energy $k^{+}=\frac{k}{u_{\tau}^{2}}$ are plotted (Figs. 4 and 5). 


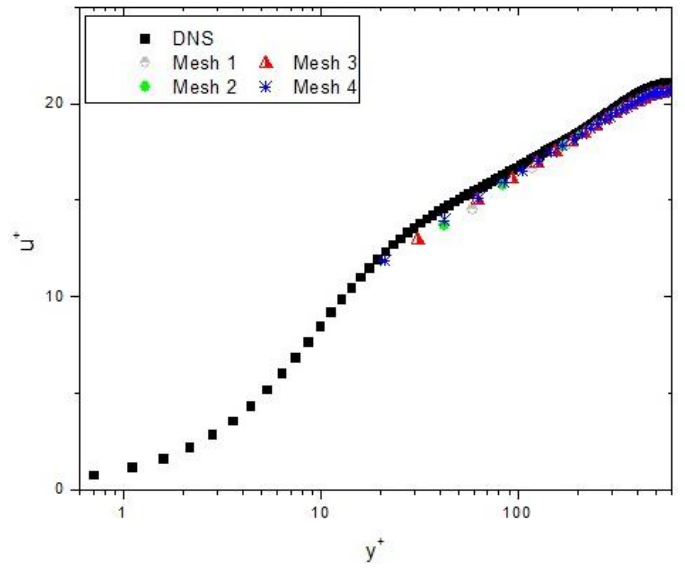

(a)

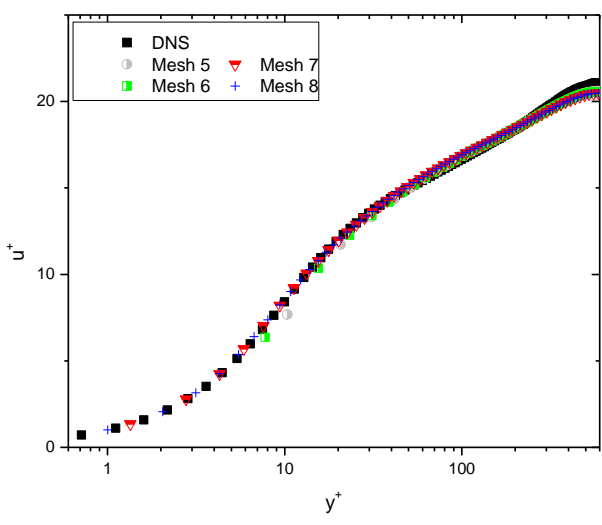

(b)

Fig.4. Comparison of non-dimensional mean streamwise velocity profiles using (a) coarse mesh with wall function (SWF), (b) fine mesh with near-wall modeling (EWT)

The different mesh configurations and corresponding wall $y^{+}$value have significant influence on the computed non-dimensional mean streamwise velocity and turbulent kinetic energy profiles.

With wall functions, the first grid point must be in the $\log$ - law region i.e $y^{+}>30$. Figures 4 (a) and 5(a) show that mesh 3 seems to be the most appropriate. As a test case, Fig. (6) presents turbulent kinetic energy (TKE) and mean velocity profiles obtained by standard wall function and first grid point at $y^{+}<30\left(y^{+} \approx 10\right.$ for mesh 5 and $y^{+} \approx 1$ for mesh 7). It shows clearly that it is impossible to obtain correct predictions when we use fine mesh with wall functions. As illustrate in Figure 6, the distribution of $u^{+}$and $k^{+}$are significantly affected. Because wall functions use the assumption of local equilibrium, that is not valid in the viscous affected region i.e $y^{+}<30$.

With near wall modeling the first grid point must be in viscous sublayer i.e $y^{+} \approx 1$. Figures 4 (b) and 5(b) show that mesh 7 seems to be the most appropriate. The viscosity affected near-wall region is completely resolved. According to user's guide of fluent (Fluent, 2005), with this modeling, we should have at least 10 cells within the viscosity affected near-wall region to be able to resolve the mean velocity and turbulent quantities in that region.

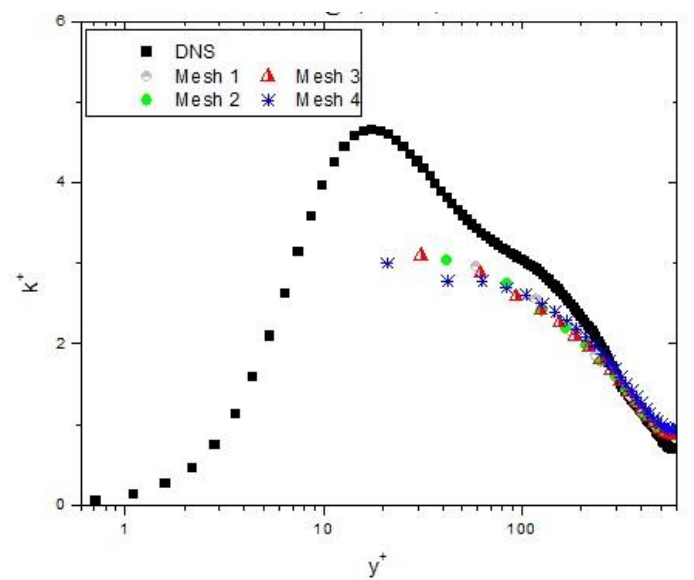

(a)

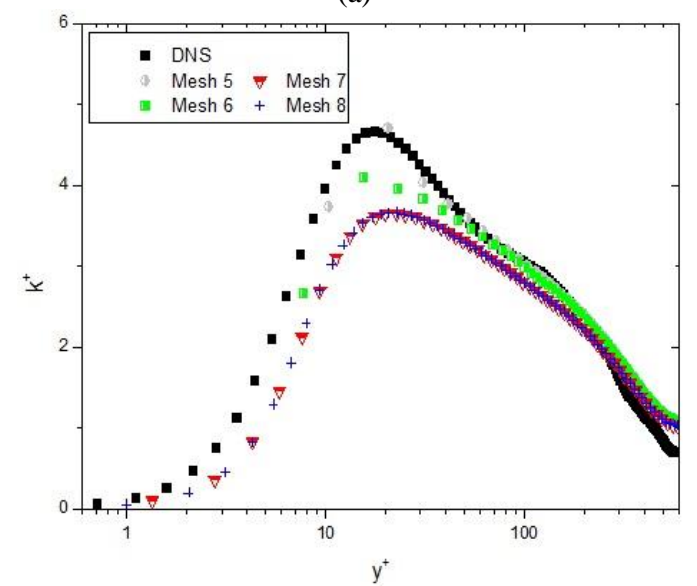

(b)

Fig. 5. Comparison of non-dimensional turbulent kinetic energy profiles using (a) coarse mesh with wall function (SWF), (b) fine mesh with near-wall modeling (EWT).

\section{B. Selection of more appropriate near wall treatment}

All different near wall treatments available in Fluent were tested: Standard wall function "SWF", Non equilibrium wall function "NEWF" and Enhanced wall treatment "EWT".

Results of mean streamwise velocity $u^{+}$and turbulent kinetic energy $k^{+}$profiles are presented in Figs. (7) and (8).

For the two test cases, channel flow and room air distribution, a fine mesh (respectively $500 \times 57$ and $45 \times 38$ ) was used for enhanced wall treatment "EWT", while a coarse mesh (respectively $500 \times 19$ and $45 \times 12$ ) was used for standard wall function "SWF" and nonequilibrium wall function "NEWF".

For the first test case (plane channel flow), Fig. 7 presents simulation results: mean streamwise velocity $u^{+}$ (Fig. 7(a)) and TKE $k^{+}$(Fig. 7(b)) profiles, with DNS data of Moser et al. (1999) for $R e_{\tau}=590$. 


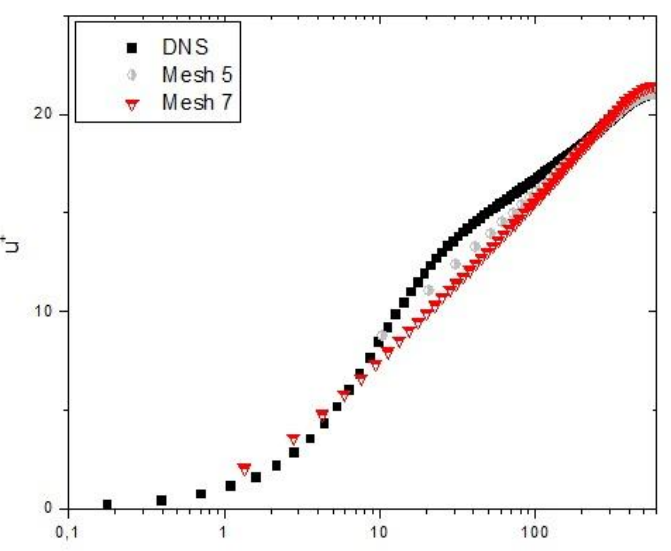

(a)

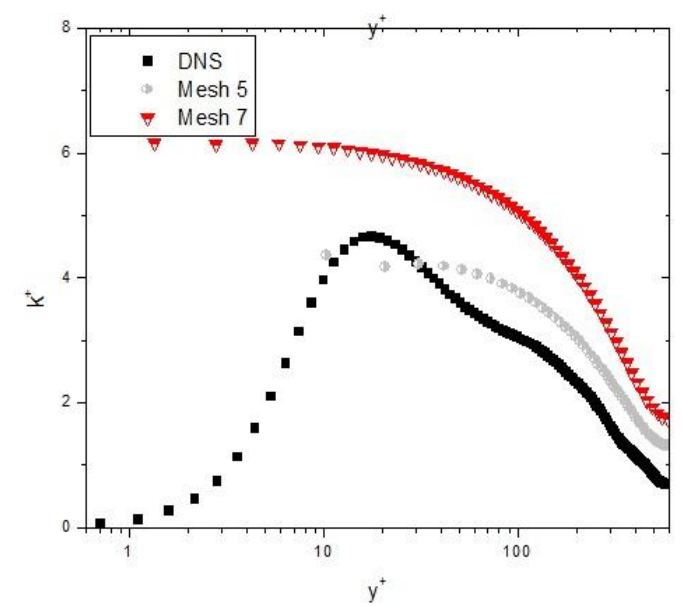

(b)

Fig. 6. Standard wall function using fine mesh (a) non-dimensional mean stream wise velocity profiles (b) non-dimensional turbulent kinetic energy profiles

On the one hand, standard "SWF" and non equilibrium "NEWF" wall functions need a coarse mesh (Fig. 1.a). The first node should be at $y^{+}>30$. Figure (7) shows that standard "SWF" and Non equilibrium "NEWF" wall functions predict well velocity profiles for $y^{+}>30$ and TKE profiles for $y^{+}>60$.

However, these near wall treatments are not able to provide details about velocity and TKE in the viscous and buffer layers. If these treatments are used, it is possible to provide an accurate description of TKE by an analytical equation and velocity by solving an ordinary differential equation "ODE" (Absi, 2009). These treatments could be therefore associated to this simple and efficient analytical method.

On the other hand, enhanced wall treatment "EWT" needs a finest mesh in the viscous sublayer (Fig. 1(b)).

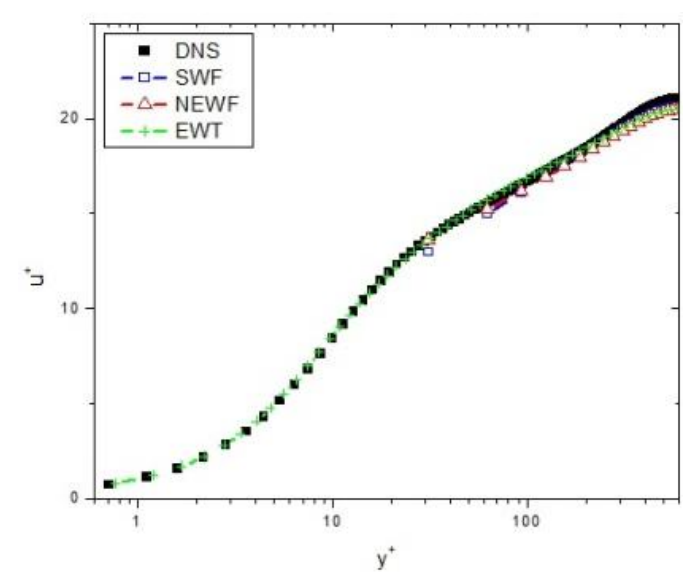

(a)

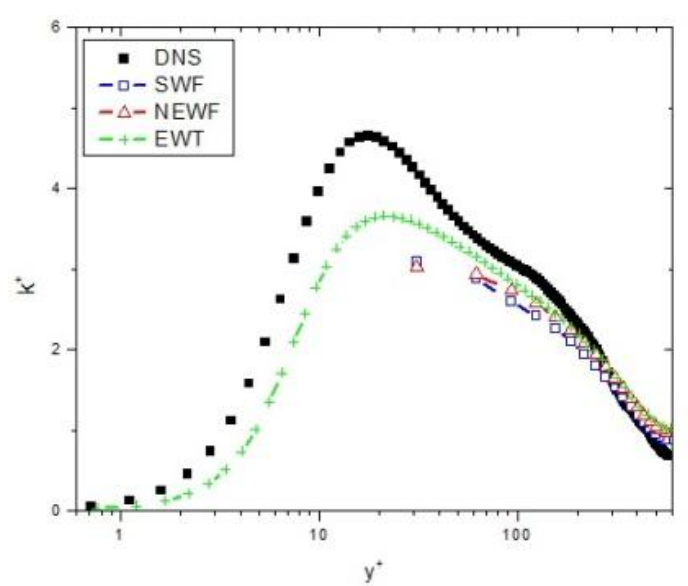

(b)

Fig. 7. comparison between predicted profiles using standard $k-\varepsilon$ model with different wall treatments and DNS data for test case 1 plane channel flow. (a) mean stremwise velocity, (b) turbulent kinetic energy

The first node should be at about $y^{+} \approx 1$. Figure (7) shows that the velocity profile is more accurate and well predicted even in the viscous and buffer layers than that standard "SWF" and non equilibrium "NEWF" wall functions. However, TKE is underestimated (Fig. 7(b)). This has no effect on velocity profile but can provide an underestimated eddy viscosity/diffusivity which could be involved in predicted particles concentrations.

In order to investigate the effect of standard k- $\varepsilon$ model on the TKE profile which is underestimated by "EWT" (Fig. 7.b), a comparison with an advanced RANS models; Re-Normalisation Group "RNG" k- $\varepsilon$ model (Yakhot et al. 1992), is done.

Figure (8) shows that RNG $k-\varepsilon$ model provides a very small improvement for velocity and TKE. Since the difference is negligible, the underestimation of TKE seems therefore not related to the used turbulence model but associated to the near wall treatment. 


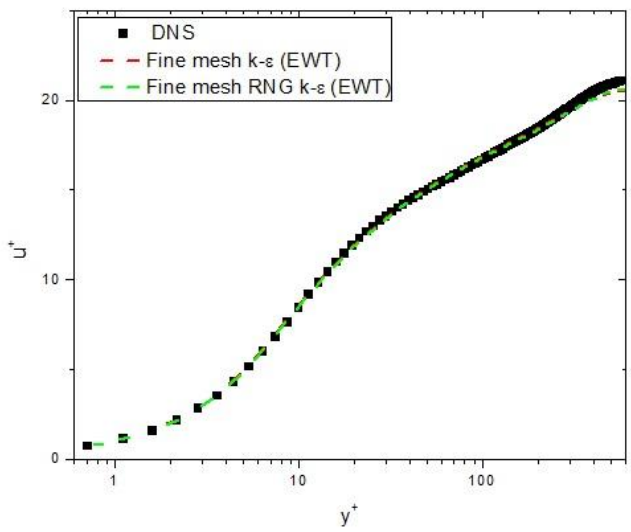

(a)

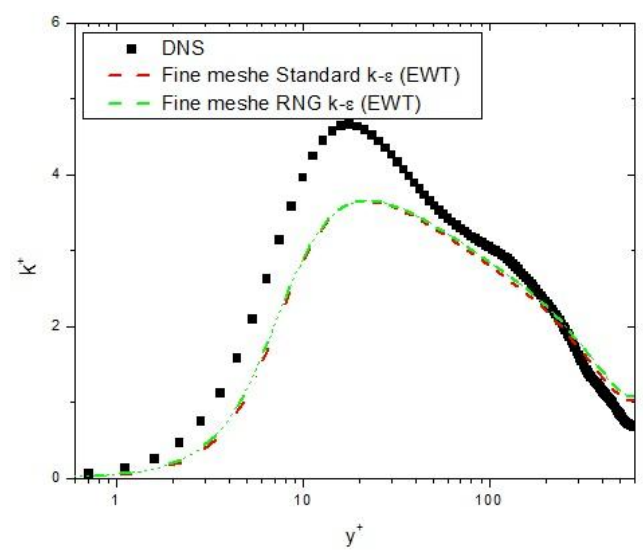

(b)

Fig. 8. Comparison between predicted profiles using standard and RNG k- $\varepsilon$ models with enhanced wall treatmant "EWT" and DNS data for test case 1 plane channel flow. (a) mean stremwise velocity, (b) turbulent kinetic energy

The second test case (benchmark test for a room air distribution), presents simulation results: mean velocity $u^{+}$(Fig. 9(a) and 9(c)) and turbulence intensity (Fig. 9(b) and $9(\mathrm{~d})$ ), with experimental data obtained by laserDoppler anemometry (Nielsen, 1990).

Figures 9(a) and 9(c) present mean velocity $u^{+}$ respectively at $x=3 \mathrm{~m}(1 / 3 \mathrm{~L})$ and $x=6 \mathrm{~m}(2 / 3 \mathrm{~L})$ while Figs. 9(b) and 8 (d) present turbulence intensity $u$ ' respectively at $x=3 \mathrm{~m}$ and $x=6 \mathrm{~m}$. Figures (9) show that for $0<y / H<0.2$ and $0.8<y / H<1$, wall functions ("SWF" and "NEWF") didn't provide values, only "EWT" provides results. This is due to the required mesh and first near wall node.

Predicted mean velocity profiles with the different nearwall treatments are quite similar (Figs. 9(a), 9(c)) for 0.2 $<y / H<0.8$. However, "EWT" provides velocities near the walls (where wall functions are unable to provide values) but needs more computation time.

More important scatter is shown for RMS fluctuation velocities at $x=3 \mathrm{~m}$ (Fig. 9(b)). All near-wall treatments fail to predict RMS fluctuation velocities for 0.2 $<y / H<0.5$. NEWF seems to be the less accurate. In contrast, at $x=6 \mathrm{~m}$ (Fig. 9(d)) wall functions seem more accurate for $0.6<y / H<0.8$.

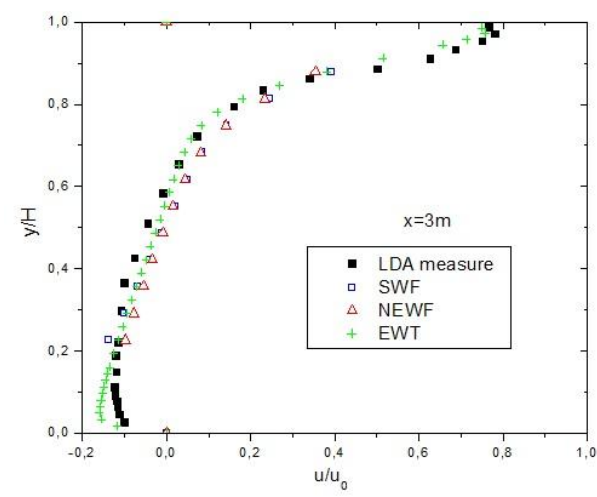

(a)

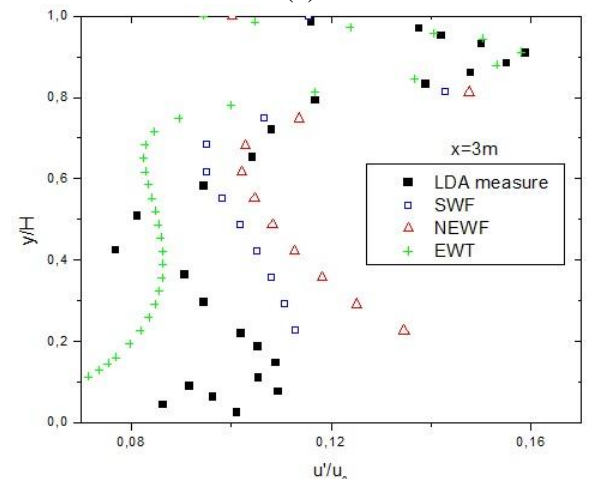

(b)

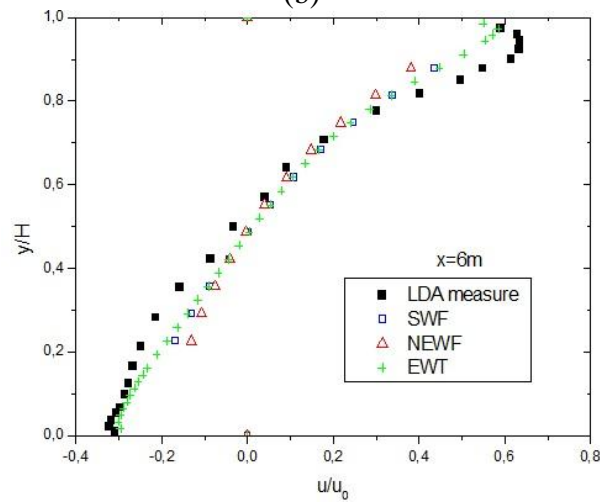

(c)

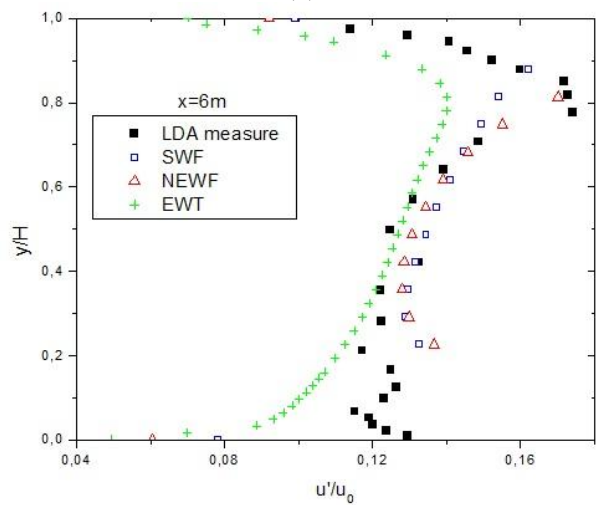

(d)

Fig. 9. Comparison between predicted profiles using standard $\mathrm{k}-\varepsilon$ model with different wall treatments and experimental data for test case 2 benchmark test for a room air distribution. (a) mean velocity at $x=3 \mathrm{~m}$, (b) RMS fluctuation velocity at $x=3 \mathrm{~m}$, (c) mean velocity at $x=6 \mathrm{~m},(\mathrm{~d})$ RMS fluctuation velocity at $x=6 \mathrm{~m}$. 
At $\mathrm{x}=3 \mathrm{~m}$, EWT provides accurate velocity (Fig. 9(a)) and RMS (Fig. 9(b)) for $0.8<\mathrm{y} / \mathrm{H}<1$. However, it under-predicts velocity for $0<\mathrm{y} / \mathrm{H}<0.2$ (Fig. 9(a)). At the opposite, at $\mathrm{x}=6 \mathrm{~m}$ velocities obtained by EWT are well predicted for $0<\mathrm{y} / \mathrm{H}<0.2$ (Fig. 9(c)) while they are under-predicted for $0.8<\mathrm{y} / \mathrm{H}<1$. These observations suggest that the flow is well predicted near the inlet and the outlet. At $3 \mathrm{~m}$, the flow is well predicted in the upper part (inlet), while at $6 \mathrm{~m}$ the flow is better described in the lower part (outlet). This could be related to recirculation zones which are not well described in these simulations.

\section{Conclusions}

Airflow simulations with different near-wall treatments were applied to two test cases.

The first test case, is the fully developed plane channel flow, similar to a flow through a long corridor in a building, simulation results: i.e. mean stream wise velocity and TKE profiles, were compared to DNS data for $R e_{\tau}=590$. Standard "SWF" and non equilibrium "NEWF" wall functions need a coarse mesh. The first node should be at $y^{+}>30$. "SWF" and "NEWF" wall functions predict well velocity profiles for $y^{+}>30$ and TKE profiles for $y^{+}>60$. However, they are not able to provide details about velocity and TKE in the viscous and buffer layers. Enhanced wall treatment "EWT" needs a finest mesh in the viscous sublayer. The first node should be at about $y^{+} \approx 1$. Velocity profile is more accurate and well predicted even in the viscous and buffer layers. TKE is underestimated; this could provide an underestimated eddy viscosity/diffusivity and therefore could have an effect on predicted temperature and particles concentration. Simulations do not show any difference between standard and RNG $k-\varepsilon$ models. The underestimated TKE seems therefore associated to near wall treatments.

For the second test case, which is a benchmark one for a room air distribution, simulation results for mean velocity and turbulence intensity (at $x / L=1 / 3$ and $2 / 3$ ) were compared to experimental data. No values obtained for all simulations, by wall functions (SWF and NEWF) in the case of $0<y / H<0.2$ and $0.8<y / H<1$, only "EWT" provides results. This is due to the required mesh and first near wall node. Predicted mean velocity profiles with different near-wall treatments are quite similar for $0.2<y / H<0.8$. However, "EWT" provides velocities near the walls where the wall functions are unable to provide values. At $\mathrm{x}=3 \mathrm{~m}(x / L=1 / 3)$, all near-wall treatments fail to predict measured RMS velocities $u$ ' for $0.2<\mathrm{y} / H<$ 0.5 . EWT provides accurate velocity and RMS for $0.8<$ $\mathrm{y} / H<1$. However, it under-predicts velocity for $0<\mathrm{y} / H$ $<0.2$. At the opposite, for $\mathrm{x}=6 \mathrm{~m}(x / L=2 / 3)$, velocities obtained by EWT are well predicted for $0<\mathrm{y} / \mathrm{H}<0.2$ while they are under-predicted for $0.8<\mathrm{y} / \mathrm{H}<1$. These observations suggest that the flow is well predicted in the upper part at $x=3 m$ (inlet), while it is better described in the lower part at $\mathrm{x}=6 \mathrm{~m}$ (outlet). This seems to be in relation with recirculation zones, which are not well described. More advanced models with adequate near-wall treatments are needed for an efficient simulation of indoor airflow distribution. In our future work, we will access Low Reynolds Number models.

\section{REFERENCES}

Absi R. (2009). A simple eddy viscosity formulation for turbulent boundary layers near smooth walls, $C . R$. Mecanique, Elsevier, 337, 158-165.

Bahlaoui A., A. Raji, M. Hasnaoui, C. Ouardi, M. Naïmi and T. Makayssi (2011). Height Partition Effect on Combined Mixed Convection and Surface Radiation in a Vented Rectangular Cavity, Journal of Applied Fluid Dynamics, 4(1), 89-96.

Chen H. C. and V. C. Patel (1988). Near-Wall Turbulence Models for Complex Flows Including Separation. AIAA Journal, 26(6), 641-648.

Chen Q (1995). Comparison of Different $k-\varepsilon$ Models for Indoor Air Flow Computations, Numerical Heat Transfer, 28, part B, pp 353-369.

Chen Q. and Z. Jiang (1992). Significant questions in predicting room air motion, ASHRAE Transactions, 98( 1), 929-939.

Chen Q. and Z. Jiang (1992). Air supply method and indoor environment, Indoor environment, 1, 88102

El Gharbi N. (2007). Modélisation et simulation du transfert de chaleur et de masse dans un espace confiné : application au conditionnement d'air d'une salle de chirurgie, mémoire de magistère en physique option Energétique et Mécanique des Fluides, USTHB, Alger.

El Gharbi N., Benzaoui A., Khalil E.E., Kameel R., (2012). Analysis of indoor air quality in surgical operating rooms using experimental and numerical investigations, Mechanics \& Industry, 13(2), 123126.

Fluent Inc. (2005). Fluent 6.2 user's guide.

Haghighat F, Z. Jiang, J. C. Y. Wang and F. Allard (1992). Air Movement in Buildings Using Computational Fluid Dynamics, Transactions of the ASME, 114, 84-92.

Jongen T. (1992). Simulation and modeling of turbulent incompressible flows, PhD thesis, EPF Lausanne, Lausanne, Switzerland.

Kader B. (1993). Temperature and Concentration Profiles in Fully Turbulent Boundary Layers. Int. J. Heat Mass Transfer, 24(9), 1541-1544.

Kim S.E. and D. Choudhury (1995). A Near-Wall Treatment Using Wall Functions Sensitized to Pressure Gradient, ASME FED, 217, Separated and Complex Flows, ASME.

Launder B. E. and D. B. Spalding (1974). The Numerical Computation of Turbulent Flows, Computer Methods in Applied Mechanics and Engineering, 3, 269-289. 
N. El Gharbi et al. / JAFM, Vol. 5, No. 4, pp. 63-70, 2012.

Moser R.D., J. Kim, N. N. Mansour (1999). Direct numerical simulation of turbulent channel flow up to Re $\tau=590$, Phys. Fluids, 11(4), 943-500.

Murakami, S., S. Kato and Y. Suyama (1987). ThreeDimensional Numerical Simulation of Turbulent Airflow in a Ventilated Room by Means of a TwoEquation Model, ASHRAE Transactions, 93(2), 621-641.

Nielsen P. V. (1989). Numerical prediction of air distribution in rooms, ASHRAE, Building systems: room air and air contaminant distribution.

Nielsen P. V. (1990). Specification of a twodimensional test case, the University of Aalborg, ISSN 0902-7513 R9040.

Spalding, D. B. (1961). A single formula for the law of the wall, Trans. ASME., J. Appl. Mech, 28, 444458.

Sumon S., H. M. Arif, H. Zakir and I. Sadrul (2008), Mixed Convection in an Enclosure with Different Inlet and Exit Configurations, Journal of Applied Fluid Dynamics, 1(1), 78-93.

Weathers, J. W. (1992). A Study of Computational Fluid Dynamics Applied to Room Airflow, M S Thesis, Oklahoma State University.

Wolfstein M. (1969). The Velocity and Temperature Distribution of One-Dimensional Flow with Turbulence Augmentation and Pressure Gradient. Int. J. Heat Mass Transfer, 12, 301-318.

Yakhot V., Orszag, S.A., Thangam S., Gatski T.B., Speziale C.G., (1992). Development of turbulence models for shear flows by a double expansion technique, Physics of Fluids A, 4(7), 1510-1520. 